\title{
Automated pulse discrimination of two freely-swimming weakly electric fish and analysis of their electrical behavior during a dominance contest
}

\author{
Rafael T Guariento ${ }^{\mathrm{a}, *}$, Thiago S Mosqueiro ${ }^{\mathrm{b}}$, Paulo Matias ${ }^{\mathrm{a}}$, \\ Vinicius B Cesarino ${ }^{\mathrm{a}}$, Lirio OB Almeida ${ }^{\mathrm{a}}$, Jan FW Slaets ${ }^{\mathrm{a}}$, Leonardo P Maia ${ }^{\mathrm{a}}$, \\ Reynaldo D Pinto ${ }^{\mathrm{a}}$ \\ ${ }^{a}$ São Carlos Institute of Physics, University of São Paulo, PO Box 369, 13560-970, São \\ Carlos, SP, Brazil. \\ ${ }^{b}$ BioCircuits Institute, University of California San Diego, CA, USA
}

\begin{abstract}
Electric fishes modulate their electric organ discharges with a remarkable variability. Some patterns can be easily identified, such as pulse rate changes, offs and chirps, which are often associated with important behavioral contexts, including aggression, hiding and mating. However, these behaviors are only observed when at least two fish are freely interacting. Although their electrical pulses can be easily recorded by non-invasive techniques, discriminating the emitter of each pulse is challenging when physically similar fish are allowed to freely move and interact. Here we optimized a custom-made software recently designed to identify the emitter of pulses by using automated chirp detection, adaptive threshold for pulse detection and slightly changing how the recorded signals are integrated. With these optimizations, we performed a quantitative analysis of the statistical changes throughout the dominance contest with respect to Inter Pulse Intervals, Chirps and Offs dyads of freely moving Gymnotus carapo. In all dyads, chirps were signatures of subsequent submission, even when they occurred early in the contest. Although offs were observed in both dominant and submissive fish, they were substantially more frequent in submissive individuals, in agreement with the idea from previous studies that offs are electric cues of submission. In general, after the dominance is established the submissive fish significantly changes its average pulse rate, while the pulse rate of the dominant remained unchanged. Additionally, no chirps or offs were observed when two fish were manually kept in direct physical contact, suggesting that these electric behaviors are not automatic responses to physical contact.
\end{abstract}

Keywords: Electrocommunication, Chirp Detection, Dominance Contest, Gymnotus, Pulse Type Electric fish, Pulse Discrimination

\footnotetext{
*Corresponding author. Tel.: +55 16 33738090; Fax: +55 1633739879

Email address: rafael.tuma.guariento@usp.br (Rafael T Guariento)
} 


\section{Introduction}

Social hierarchies are established and maintained by a broad range of dynamic behaviors expressed by animals during communication (Lorenz, 1981). Weakly electric fish in the genus Gymnotus are territorial and show marked changes in their motor and electrical behavior once a dominance hierarchy is established (Batista et al., 2012; Westby, 1975a,b). The dominant fish actively swims and explores the whole environment, while the submissive fish often remains still. Sequences of Inter Pulse Intervals (IPI) reveal highly variable patterns that are clearly distinct before and after the dominance contest (Batista et al., 2012; Westby, 1975a; Zubizarreta et al., 2015). Two critical tasks are performed by using self- and conspecifc-generated electrical pulses and their feedback on fish's electrosensory system: electrolocation and electrocommunication (Black-Cleworth, 1970; Caputi et al., 2008; Castello et al., 2000; von der Emde, 2013). Detecting distortions on the stereotyped self-generated electric pulse provides information from the environment(Jun et al., 2012; Pereira and Caputi, 2010), while IPI patterns are likely used for communication among conspecifics (Forlim and Pinto, 2014).

While fish are contesting dominance, they may stop emitting pulses during variable time intervals ("offs") (Westby, 1975a,b). In some situations, instead of the stereotyped pulses, these fish can emit small electric field oscillations, known as "chirps" (Figure 2) (Batista et al., 2012; Zubizarreta et al., 2015). Offs and chirps are often related to submission (Batista et al., 2012), physical aggression and retreat. Therefore, they might be important flags used by fish to convey submission or stress. There is strong evidence that electrocommunication has an important role in dominance definition and maintenance (McGregor and Westby, 1992; Westby, 1975a).

However, to assess electrocommunication, one of the main challenges is to discriminate pulses from freely interacting fish (Black-Cleworth, 1970; Letelier and Weber, 2000; McGregor and Westby, 1992). Two distinct but electrically coupled aquaria have been used to avoid this problem (Forlim and Pinto, 2014), but complex behaviors such as chirps have never been detected with such artificial setups, possibly due the lack of behavioral cues other than the electric pulses (e.g.,movement and bites).

Here we report on improving a state-of-art classification technique (Matias et al., 2015), that discriminates pulses from Gymnotus carapo dyads, to allow automated chirp detection. We used a supervised learning algorithm, and its training required only short samples of time series with and without chirps. We applied these tools to analyze the electrical interactions during and after dominance interaction. Fish dominance roles were identified by observing behavioral cues. We analyzed data from several dyads, and discuss the changes found in the distributions of IPIs, offs and chirps, during their dominance contest. Because chirps often occur when both fish engage in close-range physical contact, we tested the hypothesis that chirps and offs could be automatically generated by the contact of electric organs when fish are touching each other. However, no chirps nor offs were observed when two fish were manually placed in physical 
contact, with their skins touching. This suggests that instead of related to an automatic mechanism, chirps and offs might be used to communicate important information that shapes the dominance context.

All our software is freely available (Matias and Guariento, 2016). Data from one of our dyads is also available (Guariento et al., 2016).

\section{Materials and Methods}

\subsection{Ethics statement}

All experimental protocols and procedures were in accordance with the ethical principles of the Society for Neuroscience and were approved by the Committee on Ethics in Animal Experimentation of the São Carlos Institute of Physics - University of São Paulo.

\subsection{Subjects and housing}

Experiments were conducted on 6 healthy adult specimens of Gymnotus carapo, $15-25 \mathrm{~cm}$ long, regardless of sex. The home tanks and feeding protocol were previously described (Forlim and Pinto, 2014). All specimens were acquired from local commercial suppliers within 15 days before experiments. Four specimens were acquired on September/October 2015 and two on June 2016.

\subsection{Experimental setup}

The experiments with freely interacting fish were performed in a glass aquarium $(100 \times 50 \times 50 \mathrm{~cm})$ filled with tap water, and shielded by a grounded metallic mesh (Faraday cage). To induce interaction between pairs of fish, no objects were placed in the measurement aquarium, leaving no spots for hiding. The water was set at room temperature $(23 \pm 2)^{\circ} \mathrm{C}$ and the conductivity was measured before and after the experiments as $(55 \pm 5) \mu \mathrm{S} / \mathrm{cm}$. The fish were placed in this setup only during the experiments.

The Electric Organ Discharges (EODs) were measured using a three-dimensional array of 12 electrodes, each consisting of a $0.2 \mathrm{~mm}$ diameter stainless steel wire (Figure 1). The electrodes were inserted through the vertices and in the mid edge of the longer sides of the measurement aquarium. Time series of 11 electrodes were differentially amplified (100 times - Texas Instruments Operational Amplifier TL07X series on inverting mode with a $10 \mathrm{~Hz}$ input high-pass filter) with a single common reference electrode, and digitized at $45.5 \mathrm{kHz}$ by a commercial acquisition system (Digidata 1322A, Molecular Devices).

\subsection{Time series from isolated fish and training protocol}

To collect enough data to calculate pulse shape statistics over a wide range of positions, each fish was placed alone in the measurement aquarium to record their own EOD time series. Each fish was left freely swimming for 10 minutes and then induced to swim for another 10 minutes, by prodding the fish with a non-conductive net. These time series were then used as training examples for a protocol based on state-of-art machine learning techniques (Matias et al., 2015), as described in section 2.7. 


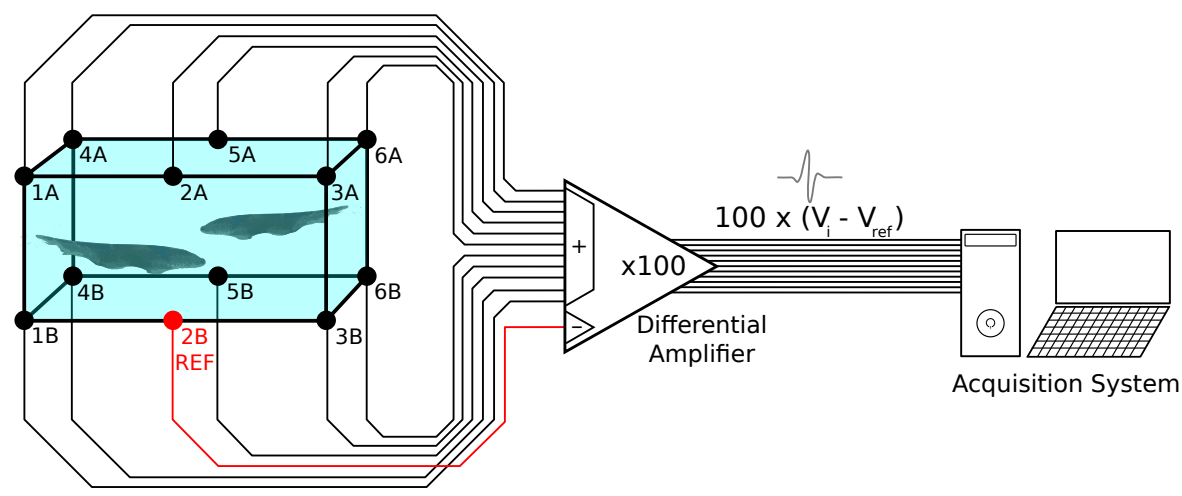

Figure 1: The fish are allowed to move freely and interact in the measurement aquarium, where the discharges from their electric organ were recorded. The time series from 11 electrodes were differentially amplified 100 times, using one electrode as reference (red wire in the figure), and then acquired by a commercial analog-to-digital converter at $45.5 \mathrm{kHz}$.

\subsection{Dominance contest}

Each pair of fish was placed simultaneously in the measurement aquarium to interact for 70min, when a contest for dominance happened, and then, moved back to their home tanks. All the experiments were performed at night (2000 - 0200h) in the dark. According to classical signatures described in the literature (Batista et al., 2012; Westby, 1975a; Zubizarreta et al., 2015), after each experiment a dominance relationship was formed. For instance, one of the fish in each dyad rapidly stop biting its conspecific and frequently swam away from their aggressive counterparts. These fish were later identified as submissive. The dominance contests were performed before the training protocol as defined by section 2.4 , assuring that the animals were naïve to the experimental setup.

\subsection{Chirp detection}

We used supervised learning to detect chirps in the time series (Figure 2a,b). First, we built a new time series by summing the absolute values of voltage of all electrodes. Several non-overlaping sections were uniformly selected, each one $0.5 \mathrm{~s}$ long, and manually inspected and labeled according to two classes: (i) sections with chirp and (ii) sections without chirps. Then, $N$ sections with chirp and $2 N$ without chirp were fed as training examples for a Random Forest (Breiman, 2001) classifier. To implement this classifier as a continuous chirp detector, the training examples were segmented in sampling windows of 2000 data points $(44 \mathrm{~ms})$. The accuracy of the classification was measured by 5 -fold cross validation (Bishop, 2006) and increased with $N$.

Empirically, chirps were always longer than $100 \mathrm{~ms}$, i.e. 3 moving windows of $44 \mathrm{~ms}$. To avoid misclassification of smaller regions, when applying the detector on the whole timeseries, chirp detection was only considered when 4 consecutive windows of 2000 data points (44 ms) were classified as containing chirps (Figure 

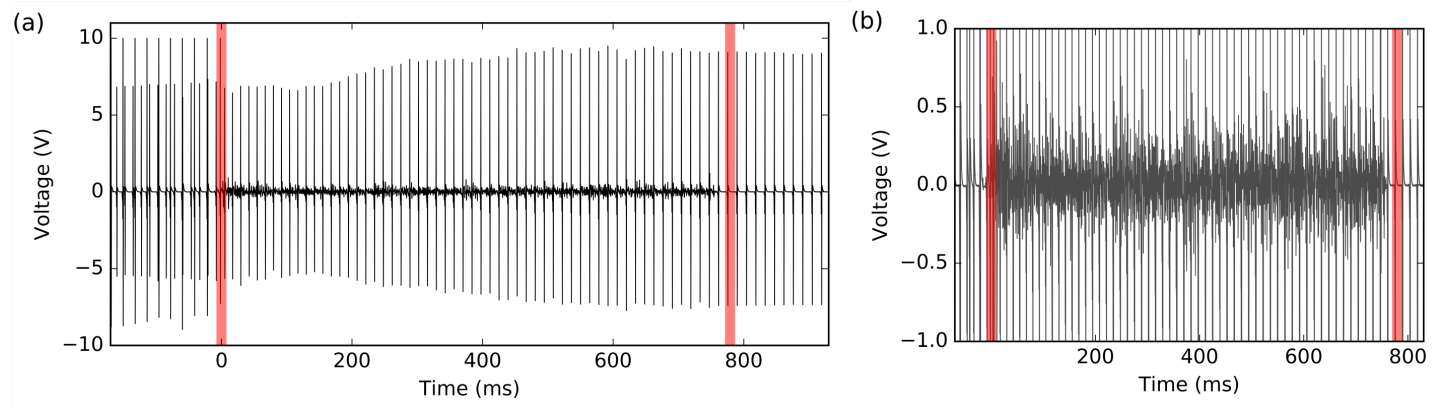

Figure 2: Time series from a single electrode containing a chirp. (a) One of the fish stops firing at $t=0$ and emits noisy-like oscillations with zero mean and amplitude ten times smaller than normal pulses (compare with $t<0$ ). (b) Zoom on chirp signal.

2c). Similarly, the end of the chirp was considered when 10 consecutive windows were classified as not containing chirps.

All chirp detection programs were written in Python and are freely available GitHub (Matias and Guariento, 2016).

\subsection{Fish discrimination}

Each pulse was assigned to a fish by using supervised learning, based on previous methodology (Matias et al., 2015) with minor improvements. Specifically, we applied Hilbert transform to the time series of all 11 electrodes, summed their absolute values, and identified peaks. In regions without chirps, the position of each pulse was then detected by finding the peaks of the summed signal with a threshold of $1 \mathrm{~V}$. In regions containing chirp, the threshold was adaptively set as $30 \%$ of the summed signals of the maximum value in the last $300 \mathrm{~ms}$. The timing of each pulse is defined when the maximum at each peak occurred. Using this method, pulse detection is independent on the fish position in the measurement aquarium (Jun et al., 2012). We also implemented a Graphical User Interface (GUI) to verify and manually correct the few pulses wrongly classified. All the discrimination programs were written in $\mathrm{C}++$ and Python, and are freely available on GitHub (Matias and Guariento, 2016).

\subsection{Statistical tests on time series of Inter Pulse Interval}

To test whether distributions of Inter Pulse Intervals (IPIs) changed throughout the dominance contest, we applied a paired Student's $t$-test on a subsampled version of the time series to avoid pseudoreplication (Lazic, 2010). IPI time series were segmented using sections of 1500 points, which represents about $18 \mathrm{~s}$ on average. Because this is larger than the average correlation length $(300 \pm 200)$, each sample is mostly uncorrelated from each other. Because the distribution of IPIs in most cases resemble a bell-shaped distribution (see Figure S6) and because failing the hypothesis of normally distributed samples induces less errors than the existence of correlation among samples (Lazic, 2010), we used a 

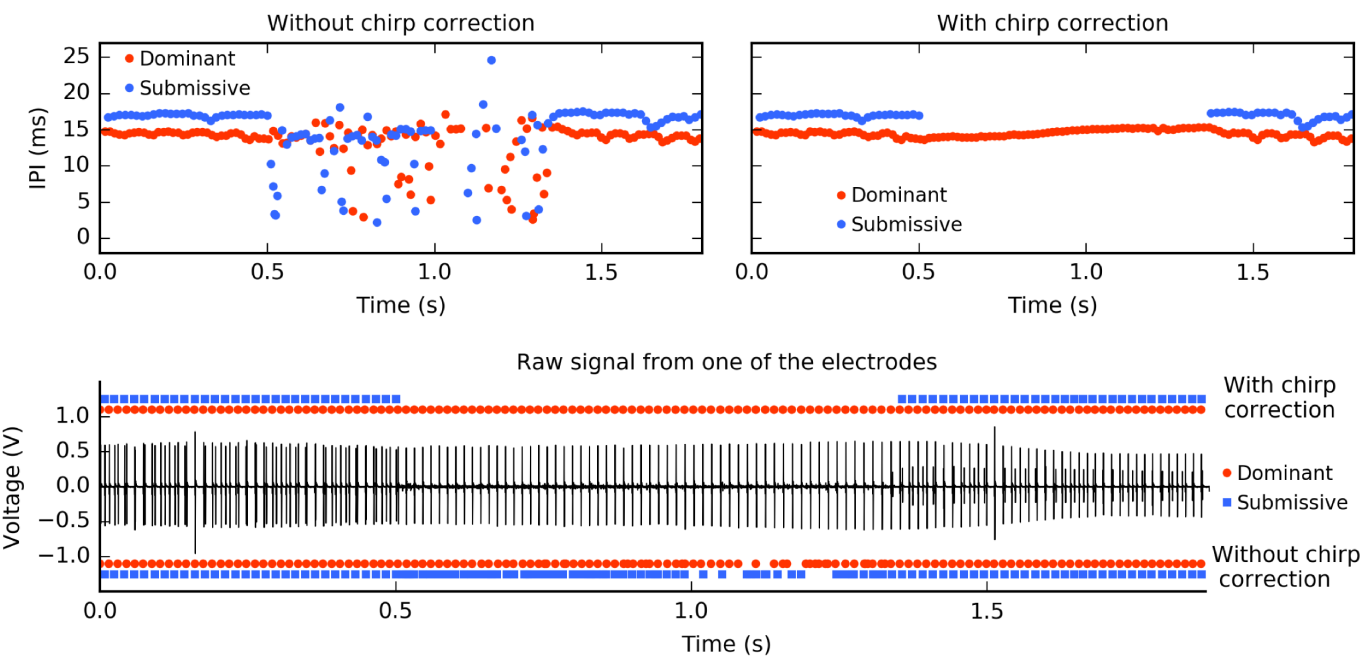

Figure 3: Fish discrimination was improved by automatically detecting chirp regions and changing the threshold for spike detection on chirp regions. Top left: Time series of Inter Pulse Intervals (IPI) before the chirp detection. Top right: Time series of Inter Pulse Intervals (IPI) of the same region after the chirp detection. Bottom: Time series acquired by one of the electrodes and pulse discrimination without (below) and with (above) chirp detection and threshold adaptation.

paired $t$-test. For completeness, we show all $t$ statistics and p-values on table S2 of Supplemental Material.

\subsection{Response of electric organs by physical contact}

To investigate whether offs and chirps are automatically generated by physical contact, causing some sort of electrical interference or short-circuiting, we picked pairs of fish and placed them by hands, until their skins touched. We repeated this experiment 4 times with distinct pairs, both using bare hands and gloves, but no difference was observed. Their bodies were positioned for $2-5$ minutes in parallel, anti-parallel and orthogonal directions (Figure 8 Top), with an interval of about 2 minutes between positions. A dipole electrode was placed near each fish to record its electric activity, using commercial apparatus (A-M Systems 1700 differential AC amplifier, Digidata 1322A, Molecular Devices).

\section{Results}

Chirps were automatically detected with $99.9 \%$ of accuracy throughout all dyads tested. The accuracy in detecting chirps saturated when the number of training examples $N$ was set to $10 \%$ of the total number of chirps present in each time series, and using a Random Forest composed of 200 decision trees. Remarkably, training data from a single dyad allowed detection of all other dyads with the same high accuracy, showing that our model learned general 

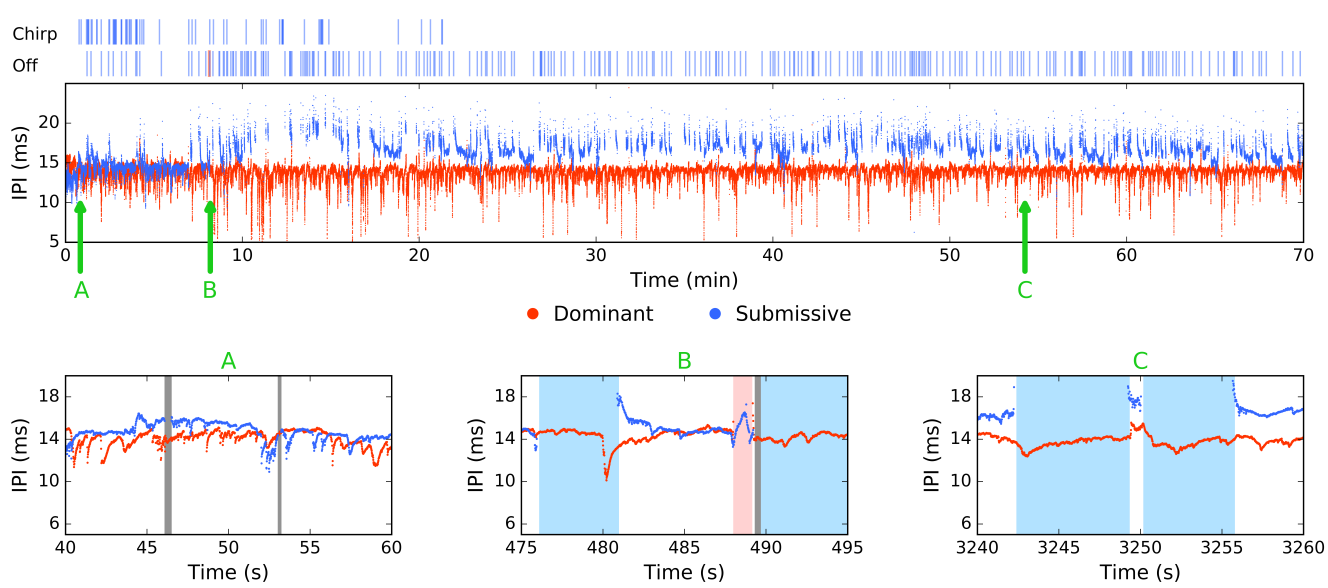

Figure 4: Time series of Inter Pulse Intervals (IPIs) with two fish freely swimming in the same aquarium. Blue represents the submissive fish and red represents the dominent fish. Top: Complete recording with bars representing the time stamps of the onset of chirps and offs. The gaps on the IPI time series are due to chirps and offs. The timestamps of each off and chirp are represented by vertical bars above the plot. Bottom: Zoom on the IPI time series. Chirps are represented as gray regions. Offs are represented as blue regions for the submissive fish and red regions for the dominant fish. Bottom A: During the early dominance contest $(t<430 s)$. Bottom B: During the dominance contest $(430 s<t<1000 s)$. Bottom C: After the dominance contest $(t>1000 s)$.

features of regions with chirps without overfitting. In regions where chirps were detected, updating the threshold for pulse detection improved the accuracy of pulse discrimination (Figure 3). Comparatively, ignoring chirps propagated spurious detections in the pulse discrimination that propagated throughout the time series. The current accuracy of the whole protocol is higher than $97 \%$ (See Supplemental Material), which allows quick manual corrections through the GUI.

Dominant and submissive fish changed their electrical behavior differently over the course of the dominance contest (Figure 4). We discriminated pulses from six different fish dyads during the first hour from their first encounter in the aquarium, with precision of $0.5 \mathrm{~ms}$ (Figure S2). The electrical behavior of both fish go through a transient dynamics and stabilize after the first 16min, as seen in Figure 4 (see also Figure S3). Because most dyads presented this transient behavior, we divided the IPIs time series in three sections: early dominance contest, usually the first 430s; the dominance contest, between 430s and 1000s; and after the dominance contest, which is after 1000s. Although arbitrary, all of our results are consistent with small changes in these thresholds. During the early dominance contest, the distributions of IPIs of both fish are very similar (Figure 5a,b). After a transient regime, when the submissive fish starts changing its behavior, the IPIs of five of the submissive fish changed significantly ( $p-$ 
(a)

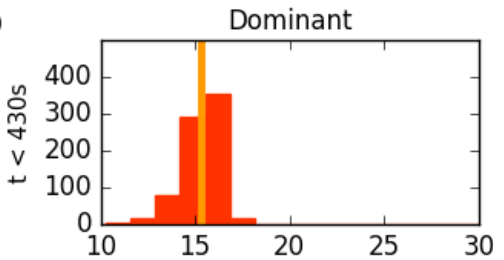

(c)

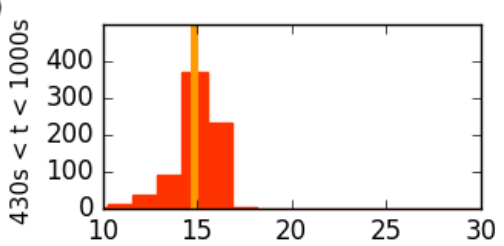

(e)

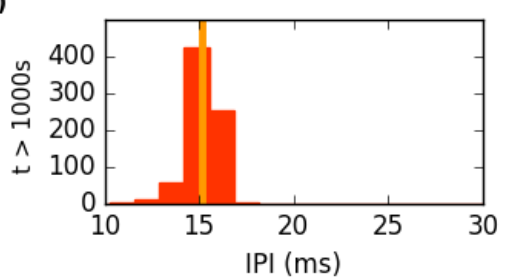

(b)

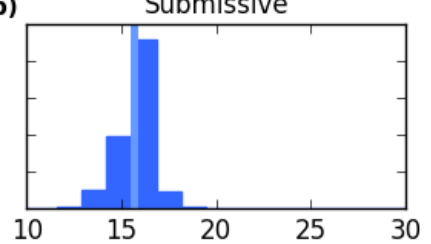

(d)

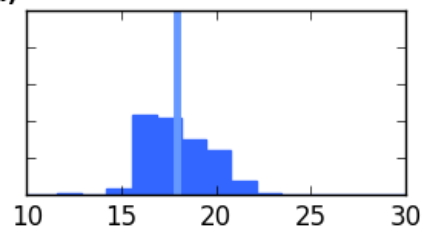

(f)

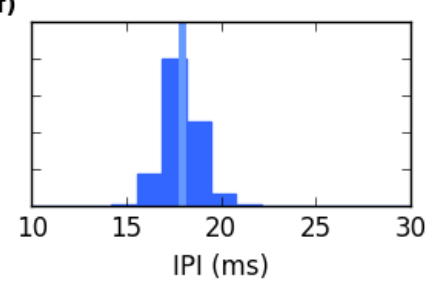

Figure 5: IPI Histograms for IPIs smaller than $30 \mathrm{~ms}$ (without chirps and offs) for dyad 1. Vertical bars denote averages. Left: No significant changes were observed for the dominant fish (Mann-Whitney U, $p>5 \%$ ). Right: Submissive fish changes to a broader distribution and eventually stabilizes at a significantly larger average (one-tail Mann-Whitney $\mathrm{U}, p<$ 2.5\%). (a) and (b): Histograms for the first 430 seconds. (c) and (d): Histograms for time between 430 and 1000 seconds. (e) and (f): Histograms for times greater than 1000 seconds.

value $\leq 0.01$ see table $\mathrm{S} 2$ ), while the average pulse rate from the dominant fish remained unchanged on three of the six dyads (Figure $5 \mathrm{c}$, d and $p$-value $\leq 0.01$ table S2). Dominant fish presented significantly less offs than submissive fish (Mann-Whitney U, $p<0.05$, see Figure S5), and no chirps were ever observed in dominant fish (Figure 6). Particularly, dyad 6, the only one recorded in 2016 (June), has some periods where both fish presented a lower pulse rate. Within these regions, no offs nor chirps were observed. Although outside these regions this dyad presents the same behavior in terms of offs and chirps as the other dyads, it is the only pair that showed these low activity regions. Due to these differences, this dyad is not included on Figure 6. However, its data and statistical results are found on Supplemental Material (Figures S3 and S6, and Tables S1 and S2).

Chirps and offs heavily skewed the distribution of IPIs of the submissive fish (Figure 7). The total duration of offs by submissive fish often represented a large portion of the whole time series (Figure 6). There were cases when the submissive fish emitted less than ten pulses between large periods of off (Figure 

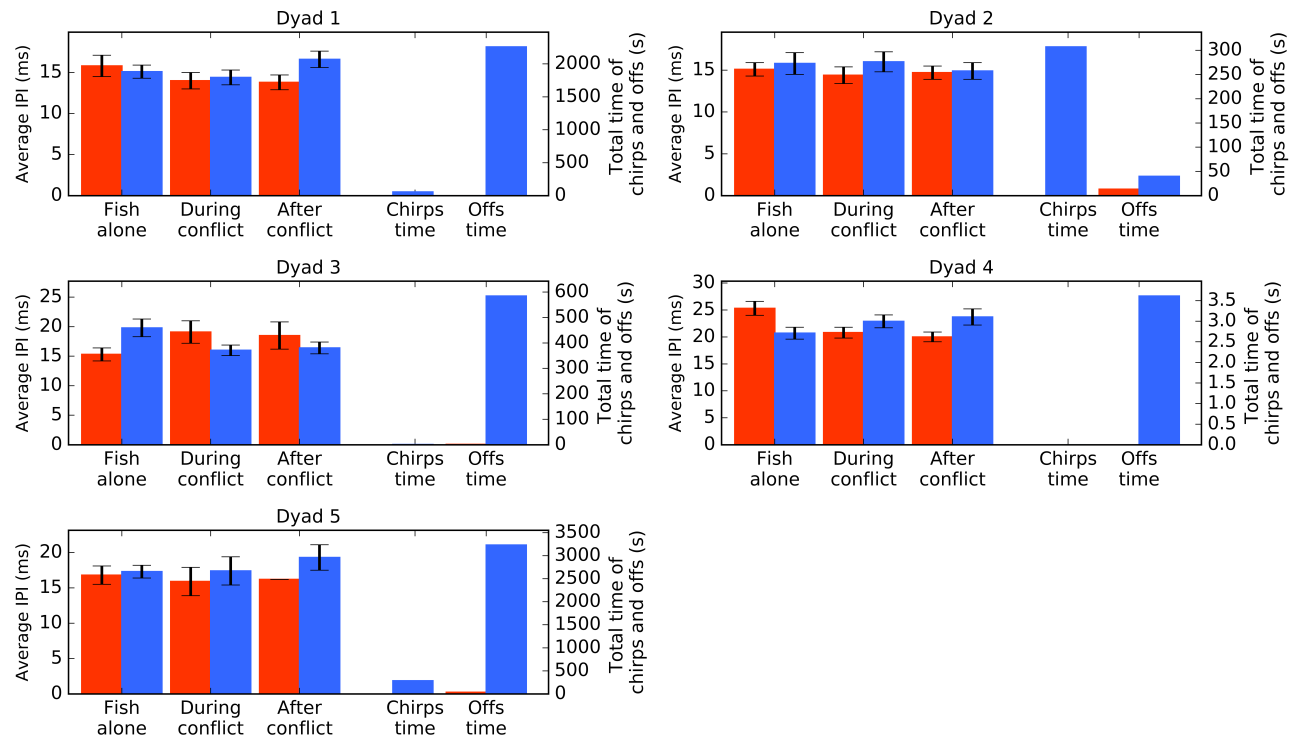

Figure 6: Average and standard deviation of Inter Pulse Intervals (IPI) and occurrences of chirps and offs for all dyads, except dyad 6 (See Supplemental Material Tables S1 and S2, and Figures S3 and S6). To not be influenced by offs and chirps, the mean and standard deviation of IPIs were measured only of IPIs shorter than $40 \mathrm{~ms}$. Offs were defined as IPIs with more than 1s. The sections "Fish alone", "During contest" and "After conflict" correspond, respectively, to the training protocol (See section 2.4), to the first 430s of the interaction between both fish, and the interaction after 1000s. Mean and standard deviation values can be found on Supplemental Material. A plot of the IPI series for each dyad can also be found on the Supplemental Material. 
(a)

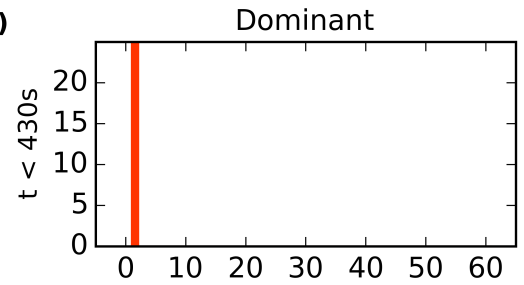

(c)

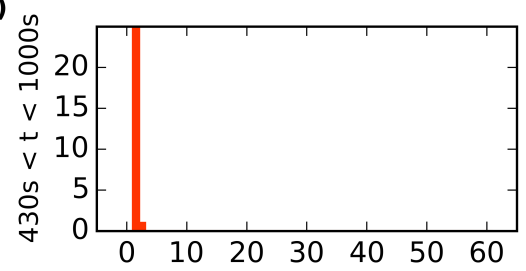

(e)

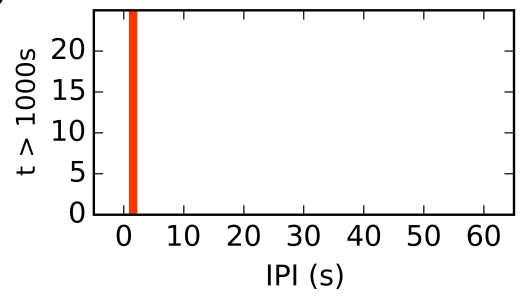

(b)

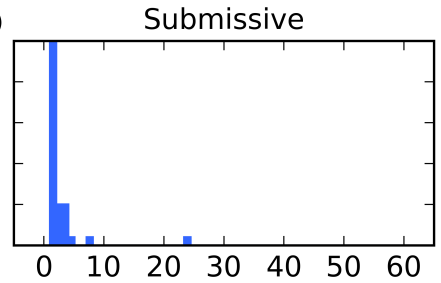

(d)

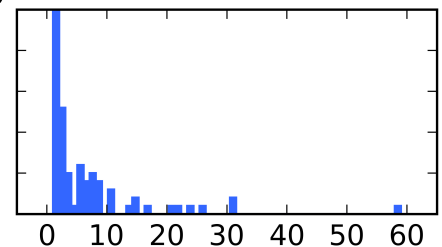

(f)

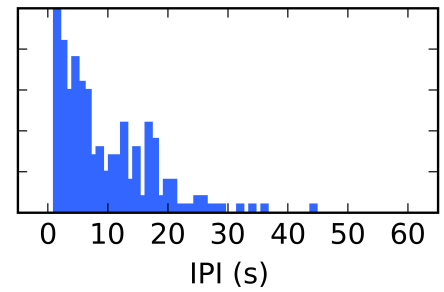

Figure 7: Inter Pulse Intervals (IPI) on a coarser time scale. Left: Dominant fish almost never shows chirps or moments of silence. Right: Distribution of IPIs of the submissive fish becomes skewed as chirps and moments of silence appear. (a) and (b): Histograms for the first 430 seconds. (c) and (d): Histograms for time between 430 and 1000 seconds. (e) and (f): Histograms for times greater than 1000 seconds.

S4 in the Supplemental Material). In some cases, the total amount of time of offs reached several minutes.

The IPI distribution when the fish is alone is always different from the distribution during and after the conflict. This shows that the dynamic changes observed in Figures 5 and 7 result from the dispute for dominance, and are not due to intrinsic characteristics of the fish.

No chirps nor offs could be observed while fish were manually placed in physical contact (Figure 8), which suggests that these behaviors are not produced automatically by direct interference of the electric organs. Oscillatory behavior on the IPI timeseries was observed regardless of their relative position (Figure 8). Interestingly, one of the fish presented chirp in the interval between manipulations, suggesting that its ability to generate chirps was not impaired by this manipulation. 


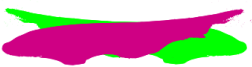

Fish in

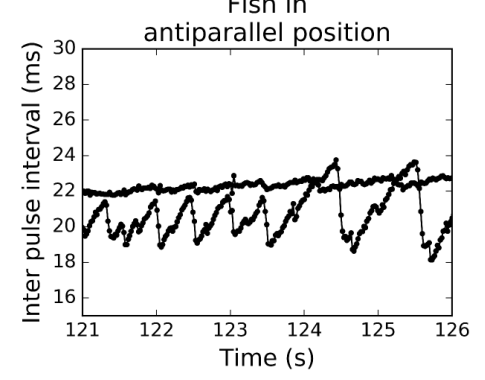

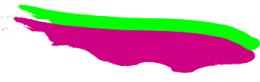

Fish kept in

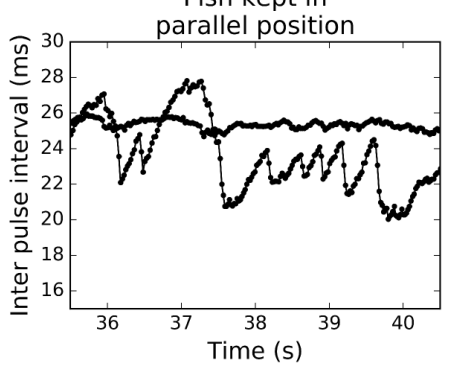

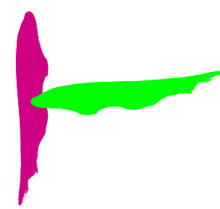

Fish kept in orthogonal position

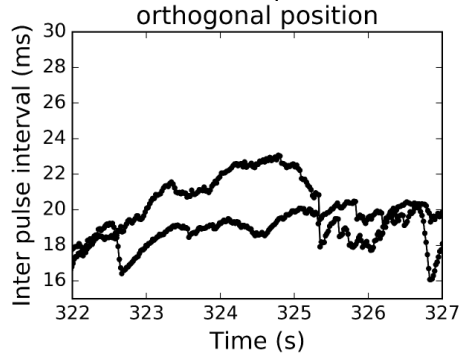

Figure 8: Fish manually held in several positions do not produce chirps when in contact. Representative sections of the IPI series are shown for each relative position. Colors were not used because no direct dominance relationship was observed in this experiment. Top: Relative positions in which the fish were kept in physical contact. Left: Fish were maintained in anti-parallel position. Center: Fish were maintained in parallel position. Right: Fish were maintained in orthogonal position.

\section{Discussion}

Electrocommunication in pulse-type electric fish (especially in many fish of the genus Gymnotus - Crampton et al. 2013) is achieved by a very rich repertoire of electrical behaviors, most of which are present only when fish are freely interacting. However, detecting and discriminating electrical activity of interacting similar fish is a challenging task if their movements are not severely restricted. We have developed a system to analyze pulse trains of two electric fish freely swimming and interacting in the same aquarium. Our system can automatically (i) detect chirps, and (ii) discriminate which fish emitted each pulse. This technique allowed us to observe how the IPI distributions of both fish changed over time in a fine-grained time scale. While the submissive fish increased its average IPI when comparing the early moments of the contest to its final, the dominant fish consistently kept its IPI rate almost unchanged (with very similar average and variance - Figure 6 The IPI time series are shown on Supplemental Material S3).

While the dominant fish maintained its pulse rate throughout the trials, Submissive fish changed significantly their electrical behavior to different average IPIs in almost all acquisitions (Figre 6), likely to avoid using the same average IPI of the dominant fish, as suggested previously (Batista et al., 2012; Westby, 1975a; Zubizarreta et al., 2015). Previous studies also suggest that electric fish change its pulse rate in order to avoid interference in electrolocation (Jamming Avoidance Response - JAR) (Max Westby, 1979). In addition, we observed signatures of JAR when the fish were manually held during the attempt to produce 
chirps (Figure 8). However, when they are freely swimming, JAR are only observed sparsely. This could be evidence that when fish are not allowed to freely interact, they express only a limited repertoire of EOD modulations, and JAR becomes more evident. Also, chirps were not observed by physical interference with the electric organs, nor with artificial stimulation of a single fish. Although the occurrence of chirps and offs may depend on other sensory and stereotypical stimuli, such as bites and specific electrical patterns that naturally occur during a dominance contest, this also suggests that these electrical behaviors can also be used to communicate important messages that shape the dominance contest and ultimately determine the dominance hierarchy.

Automating the detection of chirps proved extremely useful for pulse detection and to minimize the laborious work of visual inspection through long IPI time series. It improved the accuracy in pulse detection, avoiding the propagation of errors in regions affected by chirps. Due to the generality of the electric signature of chirps, it was possible to implement an efficient detector based on a minimal dataset consisting of manually classified samples. Remarkably, chirps in the time series of all dyads were automatically detected by using a model trained over the data of a single dyad, which greatly improved the applicability of this solution. With this methodology, long IPI time series from interacting fish can be efficiently analyzed, which is fundamental for investigating electrocommunication and complex hierarchical behaviors (Mosqueiro et al., 2016).

Although the whole process is computationally intensive, we have optimized our algorithms to allow them to run in a single off-the-shelf personal computer: detection of chirps and pulses of an one hour experiment with two fish takes a little more than one day, including manual corrections and inspection. Our code have a friendly GUI and are freely available on GitHub (Matias and Guariento, 2016). All our software is freely available (Matias and Guariento, 2016). Data from one of our dyads is also available (Guariento et al., 2016).

\section{Funding}

Authors acknowledge useful discussions with Brenno Gustavo Barbosa. This work was supported by the Brazilian agencies: Fundacão de Amparo à Pesquisa do Estado de São Paulo - FAPESP (www.fapesp.br), Coordenação de Aperfeiçoamento de Pessoal de Nível Superior - CAPES (www.capes.gov.br), and Conselho Nacional de Desenvolvimento Científico e Tecnológico - CNPq (www.cnpq.br). TS Mosqueiro acknowledges partial support from CNPq grant 234817/2014-3. The funders had no role in study design, data collection and analysis, decision to publish, or preparation of the manuscript.

\section{References}

\section{References}

Gervasio Batista, Lucía Zubizarreta, Rossana Perrone, and Ana Silva. Non-sexbiased dominance in a sexually monomorphic electric fish: Fight structure 
and submissive electric signalling. Ethology, 118(4):398-410, 2012. ISSN 14390310. doi: 10.1111/j.1439-0310.2012.02022.x. URL http://dx.doi.org/10. $1111 / j .1439-0310 \cdot 2012.02022 . x$.

Christopher M Bishop. Pattern recognition. Machine Learning, 128, 2006.

Patricia Black-Cleworth. The role of electrical discharges in the nonreproductive social behaviour of textitGymnotus carapo (Gymnotidae, Pisces). Animal Behaviour Monographs, 3:1-IN1, 1970.

Leo Breiman. Random forests. Machine Learning, 45(1):5-32, 2001. ISSN 15730565. doi: 10.1023/A:1010933404324. URL http://dx.doi.org/10.1023/A : 1010933404324 .

Angel A. Caputi, María E. Castelló, Pedro A. Aguilera, Carolina Pereira, Javier Nogueira, Alejo Rodríguez-Cattaneo, and Carolina Lezcano. Active electroreception in Gymnotus omari: Imaging, object discrimination, and early processing of actively generated signals . Journal of Physiology-Paris, 102 (4-6):256 - 271, 2008. ISSN 0928-4257. doi: http://dx.doi.org/10.1016/ j.jphysparis.2008.10.005. URL http://www.sciencedirect.com/science/ article/pii/S092842570800051X. Electrosensory Systems.

M.E. Castello, P.A. Aguilera, O. Trujillo-Cenoz, and A.A. Caputi. Electroreception in Gymnotus carapo: pre-receptor processing and the distribution of electroreceptor types. Journal of Experimental Biology, 203(21):3279-3287, 2000. ISSN 0022-0949. URL http://jeb.biologists.org/content/203/ $21 / 3279$.

W. G. R. Crampton, A. Rodríguez-Cattáneo, N. R. Lovejoy, and A. A. Caputi. Proximate and ultimate causes of signal diversity in the electric fish Gymnotus. Journal of Experimental Biology, 216(13):2523-2541, 2013. ISSN 0022-0949. doi: 10.1242/jeb.083261. URL http://jeb.biologists.org/ content/216/13/2523.

Caroline G. Forlim and Reynaldo D. Pinto. Automatic realistic real time stimulation/recording in weakly electric fish: Long time behavior characterization in freely swimming fish and stimuli discrimination. PLoS ONE, 9(1):1-14, 01 2014. doi: 10.1371/journal.pone.0084885. URL http://dx.doi.org/10. $1371 \% 2$ Fjournal . pone. 0084885 .

R.T. Guariento, T.S. Mosqueiro, P. Matias, V.B. Cesarino, L.O.B. Almeida, J.F.W. Slaets, L.P. Maia, and R.D. Pinto. Electrical recordings of two freelyswimming weakly electric fish (gymnotus sp.) in a large aquarium, 2016. URL https://figshare.com/s/8a38ce0e86eed33543d5.

James J. Jun, André Longtin, and Leonard Maler. Precision measurement of electric organ discharge timing from freely moving weakly electric fish. Journal of Neurophysiology, 107(7):1996-2007, 2012. ISSN 0022-3077. doi: 10.1152/ jn.00757.2011. URL http://jn. physiology .org/content/107/7/1996. 
Stanley E Lazic. The problem of pseudoreplication in neuroscientific studies: is it affecting your analysis? BMC neuroscience, 11(1):1, 2010.

Juan Carlos Letelier and Pamela P. Weber. Spike sorting based on discrete wavelet transform coefficients. Journal of Neuroscience Methods, 101(2):93 106, 2000. ISSN 0165-0270. doi: http://dx.doi.org/10.1016/S0165-0270(00) 00250-8. URL http://www.sciencedirect.com/science/article/pii/ S0165027000002508.

Konrad Lorenz. The foundations of ethology. Springer, New York, 1981. ISBN 978-3-211-99936-3.

P. Matias and R.T. Guariento. gymnotools, 2016. URL http://github.com/ neurobiofisica/gymnotools/.

Paulo Matias, Jan Frans Willem Slaets, and Reynaldo Daniel Pinto. Individual discrimination of freely swimming pulse-type electric fish from electrode array recordings. Neurocomputing, 153:191 - 198, 2015. ISSN 09252312. doi: http://dx.doi.org/10.1016/j.neucom.2014.11.037. URL http: //www.sciencedirect.com/science/article/pii/S0925231214015707.

G. W. Max Westby. Electrical communication and jamming avoidance betwen resting gymnotus carapo. Behavioral Ecology and Sociobiology, 4(4):381-393, 1979. ISSN 1432-0762. doi: 10.1007/BF00303244. URL http://dx.doi. org/10.1007/BF00303244.

Peter K. McGregor and G.W. Max Westby. Discrimination of individually characteristic electric organ discharges by a weakly electric fish. $A n$ imal Behaviour, 43(6):977 - 986, 1992. ISSN 0003-3472. doi: http://dx. doi.org/10.1016/S0003-3472(06)80011-4. URL http://www. sciencedirect . com/science/article/pii/S0003347206800114.

Thiago Mosqueiro, Martin Strube-Bloss, Rafael Tuma, Reynaldo Pinto, Brian H Smith, and Ramon Huerta. Non-parametric change point detection for spike trains. In Conference on Information Sciences and Systems (CISS) - IEEE, At Princeton, Volume: 50th, volume 50, page 6, 2016.

Ana Carolina Pereira and Angel Ariel Caputi. Imaging in electrosensory systems. Interdisciplinary Sciences: Computational Life Sciences, 2(4):291307, 2010. ISSN 1867-1462. doi: 10.1007/s12539-010-0049-2. URL http: //dx.doi.org/10.1007/s12539-010-0049-2.

Gerhard von der Emde. Electroreception, pages 409-425. Springer Berlin Heidelberg, Berlin, Heidelberg, 2013. ISBN 978-3-642-10769-6. doi: 10.1007/978-3-642-10769-6_19. URL http://dx.doi.org/10.1007/ 978-3-642-10769-6_19.

GW Westby. Comparative studies of the aggressive behaviour of two gymnotid electric fish (Gymnotus carapo and Hypopomus artedi). Animal behaviour, 23 
(1):192 — 213, February 1975a. ISSN 0003-3472. doi: 10.1016/0003-3472(75) 90065-2. URL http://dx.doi.org/10.1016/0003-3472(75)90065-2.

G.W.Max Westby. Further analysis of the individual discharge characteristics predicting social dominance in the electric fish, Gymnotus carapo . Animal Behaviour, 23, Part 2:249 - 260, 1975b. ISSN 0003-3472. doi: http://dx.doi. org/10.1016/0003-3472(75)90073-1. URL http://www. sciencedirect. com/ science/article/pii/0003347275900731.

Lucía Zubizarreta, Philip K. Stoddard, and Ana Silva. Aggression Levels Affect Social Interaction in the Non-Breeding Territorial Aggression of the Weakly Electric Fish, Gymnotus omarorum. Ethology, 121(1):8-16, 2015. ISSN 1439-0310. doi: 10.1111/eth.12299. URL http://dx.doi.org/10.1111/eth. 12299. 